\title{
Influence of Adsorption of Dopant on Distribution of the Dopant in A P-N-Junction
}

\author{
E.L. Pankratov ${ }^{1, *}$, E.A. Bulaeva ${ }^{2}$ \\ ${ }^{1}$ Nizhny Novgorod State University, 23 Gagarin Avenue, Nizhny Novgorod, 603950, Russia \\ ${ }^{2}$ Nizhny Novgorod State University of Architecture and Civil Engineering, 65 Il'insky Street, Nizhny Novgorod, 603950, Russia \\ *Corresponding Author: elp2004@mail.ru
}

Copyright (C) 2013 Horizon Research Publishing All rights reserved.

\begin{abstract}
It has been shown, that manufacturing diffusive- and implanted-junction rectifiers in a semiconductor heterostructure and optimization of annealing give us possibility to increase sharpness of p-n-junction and homogeneity of dopant distribution in doped area. In this paper we consider influence of adsorption of dopant in the heterostructure on distribution of dopant concentration in the p-n-junction. Based on results of modeling we show, that adsorption of dopant gives us possibility to decrease both sharpness of the $\mathrm{p}$-n-junction and homogeneity of dopant distribution in enriched by the dopant area.
\end{abstract}

Keywords Diffusive-and Implanted-Junction Heterorectifiers, Influence of Adsorption of Dopant, Optimization of Manufacturing

\section{Introduction}

One of actual questions of manufacturing of integrated circuits is decreasing of dimensions of their elements. The decreasing gives us possibility to increase density of elements in integrated circuits and to manufacture circuits with smaller thickness. One way to decrease dimensions of elements of integrated circuits is design of new technological processes [1-5]. Another way to decrease the dimensions is refinement of existing technological processes [6-9]. It has been recently introduced an approach to manufacture diffusive- and implanted-junction rectifiers in a heterostructure [10-13]. The approach with account optimization of annealing of dopant and/or radiation defects gives us possibility to decrease dimensions of elements of integrated circuits. In the present paper we consider a heterostructure with two layers, which consist of a substrate with known type of conductivity ( $p$ or $n$ ) and an epitaxial layer. A dopant has been infused or implanted in the epitaxial layer to produce reverse type of conductivity ( $n$ or $p$ ). Farther we consider annealing of radiation defects and/or dopant. One can obtain spreading of distribution of dopant concentration during the annealing. When the dopant achieves the interface between layers of heterostructure, the distribution of concentration will be distorted. The distortion under specific conditions leads to increasing of sharpness of $p$ - $n$ - junction and homogeneity of dopant distribution in enriched by the dopant area.

The considered epitaxial layer and substrate are manufactured by using different materials. Lattice spacings of the materials are different from each other. In this situation one can find mismatch-induced strain. In this situation dislocations of discrepancy could be generated, when large difference of lattice spacings are exist. Another defect of heterostructure is inaccurate external surface of the heterostructure. It could be also generated different complexes of dopants: complexes of atoms of dopant with atoms of another dopants and atoms of materials of heterostructure. Main aim of the present paper is analysis of influence of one type of defects (losing of atoms of dopants to generation of different complexes) on both sharpness of $p$ - $n$-junction, which manufactured in the heterostructure in Fig. 1, and homogeneity of dopant distribution in enriched by the dopant.

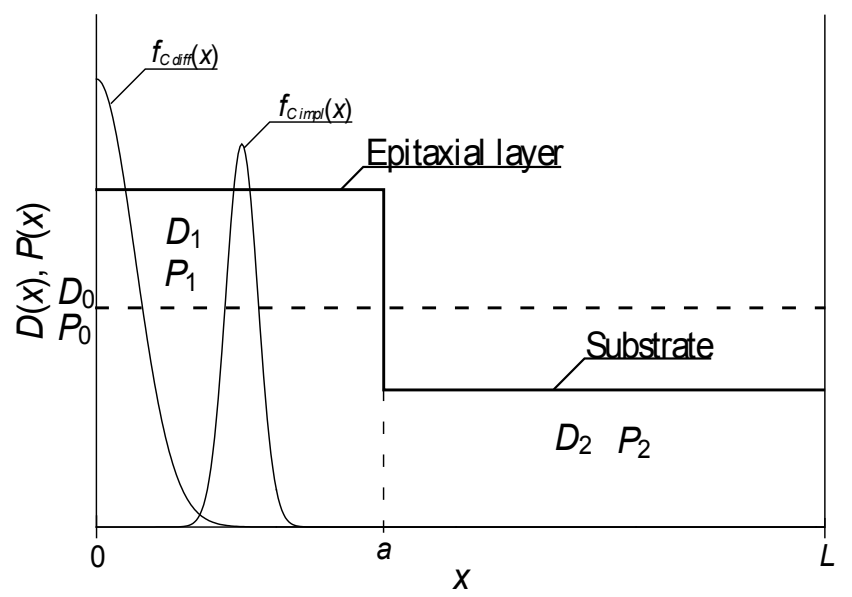

Figure 1. Heterostructure with substrate and epitaxial layer. The figure also shows initial distributions of dopant concentration for doping by diffusion and ion implantation. Here $D(x)$ and $P(x)$ are dopant diffusion coefficient and limit of solubility of dopant. $D_{i}(x)$ and $P_{i}(x), i=1,2$ are values of dopant diffusion coefficient and limit of solubility of dopant in layers of heterostructure; $D_{0}$ and $P_{0}$ are the average values of dopant diffusion coefficient and limit of solubility of dopant 


\section{Method of Solution}

To solve our aim we shall determine spatiotemporal distributions of concentrations dopant and radiation defects. We determine the distributions by solving the second Fick's low

$$
\frac{\partial C(x, t)}{\partial t}=\frac{\partial}{\partial x}\left[D_{C} \frac{\partial C(x, t)}{\partial x}\right]-k_{C}(x, T) C(x, t)(1)
$$

with boundary and initial conditions

$$
\begin{gathered}
\left.\frac{\partial C(x, t)}{\partial x}\right|_{x=0}=0,\left.\frac{\partial C(x, t)}{\partial x}\right|_{x=L}=0 \\
C(x, 0)=f_{C}(x) .
\end{gathered}
$$

Here $C(x, t)$ is the spatiotemporal distribution of dopant concentration; $D_{C}$ is the dopant diffusion coefficient; $k_{C}(x, T)$ is the adsorption coefficient; $T$ is the temperature of annealing. Value of dopant diffusion coefficient depends on properties of materials of layers of heterostructure, speed of heating and cooling of heterostructure (with account Arrhenius low). Redistribution of dopant also depends on level of doping. Dependences of dopant diffusion coefficient could be approximated by the following relation $[14,15]$

$$
\begin{aligned}
& D_{C}= \\
& D_{L}(x, T)\left[1+\xi \frac{C^{\gamma}(x, t)}{P^{\gamma}(x, T)}\right]\left[1+\varsigma_{1} \frac{V(x, t)}{V^{*}}+\varsigma_{2} \frac{V^{2}(x, t)}{\left(V^{*}\right)^{2}}\right]
\end{aligned}
$$

Here $D_{L}(x, T)$ is the spatial (due to inhomogeneity of heterostructure) and temperature (due to Arrhenius low) dependences of dopant diffusion coefficient; $P(x, T)$ is the limit of solubility of dopant; parameter $\gamma$ depends on properties of materials of heterostructure and could be integer in the interval $\gamma \in[1,3]$ [8] (concentrational dependence of dopant diffusion coefficient has been discussed in details in [14]); $V(x, t)$ is the spatiotemporal distribution of concentration of radiation vacancies; $V^{*}$ is the equilibrium distribution of radiation vacancies. It should be noted, that doping of heterostructure by diffusion did not leads to producing of radiation damage of heterostructure and parameters $\zeta_{1}$ and $\zeta_{2}$ are equal to zero.

We determine spatiotemporal distributions of concentrations of point radiation defects and their simplest complexes by solution the following systems of equations $[16,17]$

$$
\begin{gathered}
\left\{\begin{array}{l}
\frac{\partial I(x, t)}{\partial t}=\frac{\partial}{\partial x}\left[D_{I}(x, T) \frac{\partial I(x, t)}{\partial x}\right]-k_{I, V}(x, T) I(x, t) V(x, t)-k_{I, I}(x, T) I^{2}(x, t) \\
\frac{\partial V(x, t)}{\partial t}=\frac{\partial}{\partial x}\left[D_{V}(x, T) \frac{\partial V(x, t)}{\partial x}\right]-k_{I, V}(x, T) I(x, t) V(x, t)-k_{V, V}(x, T) V^{2}(x, t)
\end{array}\right. \\
\left\{\begin{array}{l}
\frac{\partial \Phi_{I}(x, t)}{\partial t}=\frac{\partial}{\partial x}\left[D_{\Phi I}(x, T) \frac{\partial \Phi_{I}(x, t)}{\partial x}\right]+k_{I, I}(x, T) I^{2}(x, t)-k_{I}(x, T) I(x, t) \\
\frac{\partial \Phi_{V}(x, t)}{\partial t}=\frac{\partial}{\partial x}\left[D_{\Phi V}(x, T) \frac{\partial \Phi_{V}(x, t)}{\partial x}\right]+k_{V, V}(x, T) V^{2}(x, t)-k_{V}(x, T) V(x, t)
\end{array}\right.
\end{gathered}
$$

with boundary and initial conditions

$$
\begin{gathered}
\left.\frac{\partial I(x, t)}{\partial x}\right|_{x=0}=0,\left.\frac{\partial I(x, t)}{\partial x}\right|_{x=L}=0,\left.\frac{\partial V(x, t)}{\partial x}\right|_{x=0}=0,\left.\frac{\partial V(x, t)}{\partial x}\right|_{x=L}=0, \\
\left.\frac{\partial \Phi_{I}(x, t)}{\partial x}\right|_{x=0}=0,\left.\frac{\partial \Phi_{I}(x, t)}{\partial x}\right|_{x=L}=0,\left.\frac{\partial \Phi_{V}(x, t)}{\partial x}\right|_{x=0}=0,\left.\frac{\partial \Phi_{V}(x, t)}{\partial x}\right|_{x=L}=0 \text { (6) } \\
I(x, 0)=f_{I}(x), V(x, 0)=f_{V}(x), \Phi_{I}(x, 0)=f_{\phi I}(x), \Phi_{V}(x, 0)=f_{\phi V}(x) .
\end{gathered}
$$

Here $\mathrm{I}(\mathrm{x}, \mathrm{t})$ is the spatiotemporal distribution of concentration of interstitials; $D_{I}(x, T)$ and $D_{V}(x, T)$ are diffusion coefficients of interstitials and vacancies; $D_{\Phi I}(x, T)$ and $D_{\Phi \nu}(x, T)$ are diffusion coefficients of diinterstitials and divacancies; terms with squares of concentrations of point radiation defects $V^{2}(x, t)$ and $I^{2}(x, t)$ correspond to generation divacancies and diinterstitials (see [16] and appropriate references in the book); $\Phi_{V}(x, t)$ and $\Phi_{I}(x, t)$ are spatiotemporal distributions of concentrations of divacancies and diinterstitials; $k_{I, V}(x, T), k_{I, I}(x, T), k_{V, I}(x, T), k_{I}(x, T)$ and $k_{V}(x, T)$ are the parameters of recombination of point defects, generation and decay of their simplest complexes. 
We determine spatiotemporal distribution of dopant concentration by using method of averaging of function correction [18] with decreased value of iteration steps [19]. Framework the approach we consider distributions of dopant concentration and concentrations of radiation defects with averaged values of diffusion coefficients $D_{0 L}, D_{0 I}, D_{0 V}, D_{0 \Phi I}, D_{0 \Phi V}$ and zero values of parameters $k_{I, I}(x, T), k_{I, I}(x, T), k_{V, I}(x, T), k_{I}(x, T), k_{V}(x, T), k_{C}(x, T)$ as initial-order approximation of decision concentrations. The zero-order approximations could be written as

$$
\begin{aligned}
& C_{1}(x, t)=\frac{2 \pi}{L} \sum_{n=1}^{\infty} F_{n C} c_{n}(x) e_{n C}(t), I_{1}(x, t)=\frac{2 \pi}{L} \sum_{n=1}^{\infty} F_{n I} c_{n}(x) e_{n I}(t), \quad V_{1}(x, t)=\frac{2 \pi}{L} \sum_{n=1}^{\infty} F_{n V} c_{n}(x) \times \\
& \times e_{n V}(t), \Phi_{I 1}(x, t)=\frac{2 \pi}{L} \sum_{n=1}^{\infty} F_{n \Phi_{I}} c_{n}(x) e_{n \Phi_{I}}(t), \quad \Phi_{V 1}(x, t)=\frac{2 \pi}{L} \sum_{n=1}^{\infty} F_{n \Phi_{V}} c_{n}(x) e_{n \Phi_{V}}(t),(7) \\
& \quad F_{n C}=\int_{0}^{L} c_{n}(u) f_{C}(u) d u \quad F_{n I}=\int_{0}^{L} c_{n}(u) f_{I}(u) d u \quad F_{n V}=\int_{0}^{L} c_{n}(u) f_{V}(u) d u \quad F_{n \Phi_{I}}=\int_{0}^{L} c_{n}(u) \times \\
& \text { where } ; \quad f_{\Phi_{I}}(u) d u ; c_{n}(x)=\cos (\pi n x / L) ; \quad \int_{0}^{L} c_{n}(u) f_{\Phi_{V}}(u) d u \quad e_{n C}(t)=\exp \left(-\pi^{2} n^{2} \frac{D_{0 C} t}{L^{2}}\right) ; \\
& e_{n I}(t)=\exp \left(-\pi^{2} n^{2} \frac{D_{0 I} t}{L^{2}}\right) ; e_{n V}(t)=\exp \left(-\pi^{2} n^{2} \frac{D_{0 V} t}{L^{2}}\right) ; e_{n \Phi_{I}}(t)=\exp \left(-\pi^{2} n^{2} \frac{D_{0 \Phi_{I}} t}{L^{2}}\right) ; \\
& e_{n \Phi_{V}}(t)=\exp \left(-\pi^{2} n^{2} \frac{D_{0 \Phi_{V}} t}{L^{2}}\right)
\end{aligned}
$$

We determine the second-order approximation and approximations with higher orders of concentrations of dopant and radiation defects framework standard iteration procedure [18,19]. Framework the procedure one shall replace the functions $C$ $(x, t), I(x, t), V(x, t), \Phi_{I}(x, t)$ and $\Phi_{V}(x, t)$ in the right sides of Eqs. (1), (4) and (5) on the sums $\alpha_{n C}+C_{n-1}(x, t), \alpha_{n I}+I_{n-1}(x, t), \alpha_{n V}+V$ ${ }_{n-1}(x, t), \alpha_{n \Phi I}+\Phi_{I n-1}(x, t), \alpha_{n \Phi V}+\Phi_{V n-1}(x, t)$ to determine the $n$-th-order approximations of the decision concentrations. Here $\alpha_{n C}$, $\alpha_{n}, \alpha_{n V}, \alpha_{n \Phi I}$, and $\alpha_{n \Phi V}$ are average values of the $n$-th-order approximations of the functions $C(x, t), \quad I(x, t), V(x, t), \Phi_{I}(x, t)$ and $\Phi_{V}(x, t)$. The replacement gives us possibility to obtain the following equations for the decision concentrations

$$
\begin{gathered}
\frac{\partial C_{2}(x, t)}{\partial t}=\frac{\partial}{\partial x}\left(D_{L}(x, T)\left[1+\varsigma_{1} \frac{V(x, t)}{V^{*}}+\varsigma_{2} \frac{V^{2}(x, t)}{\left(V^{*}\right)^{2}}\right]\left\{1+\xi \frac{\left[\alpha_{2 C}+C_{1}(x, t)\right]^{\gamma}}{P^{\gamma}(x, T)}\right\} \times\right. \\
\left.\times \frac{\partial C_{1}(x, t)}{\partial x}\right)-k_{C}(x, T)\left[\alpha_{2 C}+C_{1}(x, t)\right](8)
\end{gathered}
$$




$$
\left\{\begin{array}{l}
\frac{\partial \Phi_{I 2}(x, t)}{\partial t}=\frac{\partial}{\partial x}\left[D_{\Phi I}(x, T) \frac{\partial \Phi_{I 1}(x, t)}{\partial x}\right]+k_{I, I}(x, T) I^{2}(x, t)-k_{I}(x, T) I(x, t) \\
\frac{\partial \Phi_{V 2}(x, t)}{\partial t}=\frac{\partial}{\partial x}\left[D_{\Phi V}(x, T) \frac{\partial \Phi_{V 1}(x, t)}{\partial x}\right]+k_{V, V}(x, T) V^{2}(x, t)-k_{V}(x, T) V(x, t) .
\end{array}\right.
$$

Integration of the left and right sides of Eqs.(7)-(9) gives us possibility to obtain relations for the second-order approximations of concentrations of dopant and radiation defects

$$
\begin{gathered}
C_{2}(x, t)=\frac{\partial}{\partial x}\left(\int_{0}^{t} D_{L}(x, T)\left[1+\varsigma_{1} \frac{V(x, \tau)}{V^{*}}+\varsigma_{2} \frac{\left.V^{2}(x, \tau)\right]}{\left(V^{*}\right)^{2}}\right]\left\{1+\xi \frac{\left[\alpha_{2 C}+C_{1}(x, \tau)\right]^{\gamma}}{P^{\gamma}(x, T)}\right\}+\right. \\
\left.\quad \times \frac{\partial C_{1}(x, \tau)}{\partial x} d \tau\right)+f_{C}(x)-\int_{0}^{t} k_{C}(x, T)\left[\alpha_{2 C}+C_{1}(x, \tau)\right] d \tau \\
\left\{\begin{array}{c}
I_{2}(x, t)=\frac{\partial}{\partial x}\left[\int_{0}^{t} D_{I}(x, T) \frac{\partial I_{1}(x, \tau)}{\partial x} d \tau\right]-\int_{0}^{t} k_{I, I}(x, T)\left[\alpha_{2 I}+I_{1}(x, \tau)\right]^{2} d \tau- \\
V_{2}(x, t)=\frac{\partial}{\partial x}\left[\int_{0}^{t} D_{V}(x, T) \frac{\partial V_{1}(x, \tau)}{\partial x} d \tau\right]-\int_{0}^{t} k_{V, V}(x, T)\left[\alpha_{2 V}+V_{1}(x, \tau)\right]^{2} d \tau- \\
-\int_{0}^{t} k_{I, V}(x, T)\left[\alpha_{2 I}+I_{1}(x, \tau)\right]\left[\alpha_{2 V}+V_{1}(x, \tau)\right] d \tau+f_{V}(x) \\
\Phi_{I 2}(x, t)=\frac{\partial}{\partial x}\left[\int_{0}^{t} D_{\Phi I}(x, T) \frac{\partial \Phi_{I 1}(x, \tau)}{\partial x} d \tau\right]+\int_{0}^{t} k_{I, I}(x, T) I^{2}(x, \tau) d \tau- \\
\Phi_{V 2}(x, t)=\frac{\partial}{\partial x}\left[\int_{0}^{t} D_{\Phi V}(x, T) \frac{\partial \Phi_{V 1}(x, \tau)}{\partial x} d \tau\right]+\int_{0}^{t} k_{V, V}(x, T) V^{2}(x, \tau) d \tau- \\
-\int_{0}^{t} k_{I}(x, T) I(x, \tau) d \tau+f_{\Phi I}(x)
\end{array}\right. \\
-\int_{0}^{t} k_{V}(x, T) V(x, \tau) d \tau+f_{\Phi V}(x)
\end{gathered}
$$

We determine the average values of the second-order approximations of the decision concentrations by the following standard relations $[18,19]$

$$
\begin{gathered}
\alpha_{2 C}=\frac{1}{\Theta L} \int_{0}^{\Theta L} \int_{0}^{L}\left[C_{2}(x, t)-C_{1}(x, t)\right] d x d t \quad \alpha_{2 I}=\frac{1}{\Theta L} \int_{0}^{\Theta} \int_{0}^{L}\left[I_{2}(x, t)-I_{1}(x, t)\right] d x d t \\
\alpha_{2 V}=\frac{1}{\Theta L} \int_{0}^{\Theta L} \int_{0}^{L}\left[V_{2}(x, t)-V_{1}(x, t)\right] d x d t, \quad \alpha_{2 \Phi_{I}}=\frac{1}{\Theta L} \int_{0}^{\Theta L} \int_{0}^{L}\left[\Phi_{I 2}(x, t)-\Phi_{I 1}(x, t)\right] d x d t,
\end{gathered}
$$




$$
\alpha_{2 \Phi_{V}}=\frac{1}{\Theta L} \int_{0}^{\Theta L} \int_{0}^{L}\left[\Phi_{V 2}(x, t)-\Phi_{V 1}(x, t)\right] d x d t
$$

Substitution of the relations (7), (8a)-(10a) into relations (11) gives us possibility to obtain relations for average values of the second-order approximations of decision concentrations $\alpha_{2 C}, \alpha_{2 I}, \alpha_{2 V}, \alpha_{2 \Phi_{I}}, \alpha_{2 \Phi_{V}}$

$$
\begin{array}{r}
\alpha_{2 C}=\frac{1}{L} \int_{0}^{L} f_{C}(x) d x-\frac{1}{L \Theta} \int_{0}^{\Theta}(\Theta-t) \int_{0}^{L} k_{C}(x, T)\left[\alpha_{2 C}+C_{1}(x, t)\right] d x d t, \\
\alpha_{2 I}=\sqrt{\left(1+A_{I V 01}+A_{I I 10}+\alpha_{2 V} A_{I V 00}\right)^{2}-4 A_{I I 00}\left[\alpha_{2 V} A_{I V 10}-A_{I I 20}+A_{I V 11}-\frac{1}{L} \int_{0}^{L} f_{I}(x) d x\right]} \times \\
\times \frac{1}{2 A_{I I 00}}-\frac{1+A_{I V 01}+A_{I I 10}+\alpha_{2 V} A_{I V 00}}{2 A_{I I 00}}, \\
\alpha_{2 V}=\frac{1}{2 B_{4}} \sqrt{\frac{\left(B_{3}+A\right)^{2}}{4}-4 B_{4}\left(y+\frac{B_{3} y-B_{1}}{A}\right)}-\frac{B_{3}+A}{4 B_{4}},
\end{array}
$$

where $A_{a b i j}=\frac{1}{\Theta L} \int_{0}^{\Theta}(\Theta-t) \int_{0}^{L} k_{a, b}(x, T) I_{1}^{i}(x, t) V_{1}^{j}(x, t) d x d t, B_{4}=A_{I V 00}^{2} A_{I V 00}^{2}-2\left(A_{I V 00}^{2}-\right.$ $\left.-A_{I I 00} A_{V V 00}\right)^{2}, B_{3}=A_{I V 00} A_{I V 00}^{2}-4 A_{I V 10} A_{I I 00} A_{I V 00}^{2} 2 A_{I V 00} A_{I V 01} A_{I V 00}^{2}+A_{I V 00} A_{I I 10} A_{I V 00}^{2}+A_{I V 01} \times$ $\times A_{I V 00}^{3}+4\left[2 A_{I V 01} A_{I V 00}+2 A_{I V 00}\left(1+A_{I V 01}+A_{I I 10}\right)-2 A_{I I 00}\left(A_{I V 10}+A_{V V 10}+1\right)\right]\left(A_{I I 00} A_{V V 00}-\right.$ $\left.-A_{I V 00}^{2}\right), B_{2}=A_{I V 00}^{2}\left\{\left(1+A_{I V 01}+A_{I I 10}\right)^{2}-4 A_{I I 00}\left[A_{I V 11}-A_{I I 20}-\frac{1}{L} \int_{0}^{L} f_{I}(x) d x\right]+A_{I V 00}^{2} \times\right.$ $\left.\times A_{I V 01}^{2}+2 A_{I V 00} A_{I V 01}\left(A_{I V 00}+A_{I V 00} A_{I V 01}+A_{I V 00} A_{I I 10}-4 A_{I V 10} A_{I I 00}\right)\right\}\left\{4\left[A_{I V 01} A_{I V 00}+A_{I V 00} \times\right.\right.$ $\left.\times\left(1+A_{I V 01}+A_{I I 10}\right)-A_{I I 00}\left(A_{I V 10}+A_{V V 10}+1\right)\right]^{2}+\left[A_{I V 01}\left(1+A_{I V 01}+A_{I I 10}\right)-\left(A_{V V 20}-A_{I V 11}\right) \times\right.$ $\left.\times 2 A_{I I 00}+\frac{2}{L} A_{I I 00} \int_{0}^{L} f_{V}(x) d x+A_{I V 01}\left(1+A_{I V 01}+A_{I I 10}\right)\right] 4\left[A_{I V 00}\left(1+A_{I V 01}+A_{I I 10}\right)+A_{I V 01} \times\right.$ $\left.\left.\times A_{I V 00}-A_{I I 00}\left(A_{I V 10}+A_{V V 10}+1\right)\right]\right\}, \quad B_{1}=2 A_{I V 00} A_{I V 01}\left(1+A_{I V 01}+A_{I I 10}\right)^{2}-A_{I V 00} A_{I V 01} A_{I I 00} \times$ $\times 8\left[A_{I V 11}-A_{I I 20}-\frac{1}{L} \int_{0}^{L} f_{I}(x) d x\right]+A_{I V 01}^{2}\left(A_{I V 00}+A_{I V 00} A_{I V 01}+A_{I V 00} A_{I I 10}-4 A_{I V 10} A_{I I 00}\right)-$ $-2\left[A_{I V 01}\left(1+A_{I V 01}+A_{I I 10}\right)+\frac{2}{L} A_{I I 00} \int_{0}^{L} f_{V}(x) d x+A_{I V 01}\left(1+A_{I V 01}+A_{I I 10}\right)-\left(A_{V V 20}-A_{I V 11}\right) \times\right.$ $\left.\times 2 A_{I I 00}\right]\left[2 A_{I V 00}\left(1+A_{I V 01}+A_{I I 10}\right)+2 A_{I V 01} A_{I V 00}-2 A_{I I 00}\left(A_{I V 10}+A_{V V 10}+1\right)\right], \quad B_{0}=4 A_{I I 00} \times$ $\times A_{I V 01}^{2}\left[A_{I I 20}+\frac{1}{L} \int_{0}^{L} f_{I}(x) d x-A_{I V 11}\right]+A_{I V 01}^{2}\left(1+A_{I V 01}+A_{I I 10}\right)^{2}-\left[A_{I V 01}\left(1+A_{I V 01}+A_{I I 10}\right)-\right.$ 


$$
\begin{gathered}
\left.-2 A_{I I 00}\left(A_{V V 20}-A_{I V 11}\right)+\frac{2}{L} A_{I I 00} \int_{0}^{L} f_{V}(x) d x+A_{I V 01}\left(1+A_{I V 01}+A_{I I 10}\right)\right]^{2}, A=\sqrt{8 y+B_{3}^{2}-4 B_{2}}, \\
y=\sqrt[3]{\sqrt{q^{2}+p^{3}}-q}-\sqrt[3]{\sqrt{q^{2}+p^{3}}+q}+\frac{B_{2}}{6}, q=\frac{B_{2}^{3}}{216}+\frac{B_{0}\left(4 B_{2}-B_{3}^{2}\right)-B_{1}^{2}}{8}+\frac{B_{2}}{48}\left(2 B_{1} B_{3}-8 B_{0}\right), \\
p=\left[3\left(2 B_{1} B_{3}-8 B_{0}\right)-2 B_{2}^{2}\right] / 72, \\
\alpha_{2 \Phi_{I}}=A_{I I 20}-\frac{1}{\Theta L} \int_{0}^{\Theta}(\Theta-t) \int_{0}^{L} k_{I}(x, T) I(x, t) d x d t+\frac{1}{L} \int_{0}^{L} f_{\Phi_{I}}(x) d x, \\
\alpha_{2 \Phi_{V}}=A_{V V 20}-\frac{1}{\Theta L} \int_{0}^{\Theta}(\Theta-t) \int_{0}^{L} k_{V}(x, T) V(x, t) d x d t+\frac{1}{L} \int_{0}^{L} f_{\Phi_{V}}(x) d x .
\end{gathered}
$$

Analysis of spatiotemporal distributions of concentrations of dopant and radiation defects had been done analytically by using the second-order approximation of the concentrations framework method of averaging of function corrections with decreased quantity of iteration steps. The second-order approximation is usually enough good approximation to make qualitative analysis and to obtain some quantitative results. Results of analytical calculations had been checked by comparison with results of numerical simulations.

\section{Discussion}

In this section based on obtain in previous section relations we analyzed dynamics of redistribution of dopant and radiation defects. The typical distributions of dopant (for diffusive type of doping and ion implantation, respectively) for fixed value of annealing time and different values of difference between diffusion coefficients of dopant in layers of heterostructure are presented on Figs. 2 and 3. On the present figures solid lines are distributions of dopant without accounting dopant adsorption, dashed lines are distributions of dopant with accounting dopant adsorption in the substrate. The Figs. 2 and 3 show, that interface between layers of heterostructure gives us possibility to increase sharpness of $p-n$-junction and homogeneity of dopant distribution in enriched area. Increasing of sharpness of $p-n$-junction gives us possibility to decrease switching time of the device. Increasing of homogeneity of dopant distribution gives us possibility to decrease local overheats of material during operation of $p$ - $n$-junction or to decrease dimensions of $p-n$-junction for fixed tolerance on value of overheats. The Figs. 2 and 3 also show, that accounting of adsorption gives us possibility to decrease sharpness of $p$ - $n$-junction and quantity of dopant in the substrate.

It should be noted, that increasing annealing time leads to increasing of homogeneity of dopant distribution. The increasing of homogeneity leads to decreasing of sharp- ness of $p-n$-junction. Decreasing annealing time leads to increasing of inhomogeneity of dopant distribution. The increasing of inhomogeneity leads to increasing of overheat of the area. In this situation we shall choose so value of annealing time, which correspond to compromise between increasing of sharpness of $p$ - $n$-junction and homogeneity of dopant distribution in enriched area. To determine compromise annealing time we used recently introduce criterion [10-13]. Framework the approach we approximate real distribution of dopant by stepwise function (see Figs. 4 and 5 for doping by diffusion and ion implantation, respectively). Farther we minimize the following mean-squared error

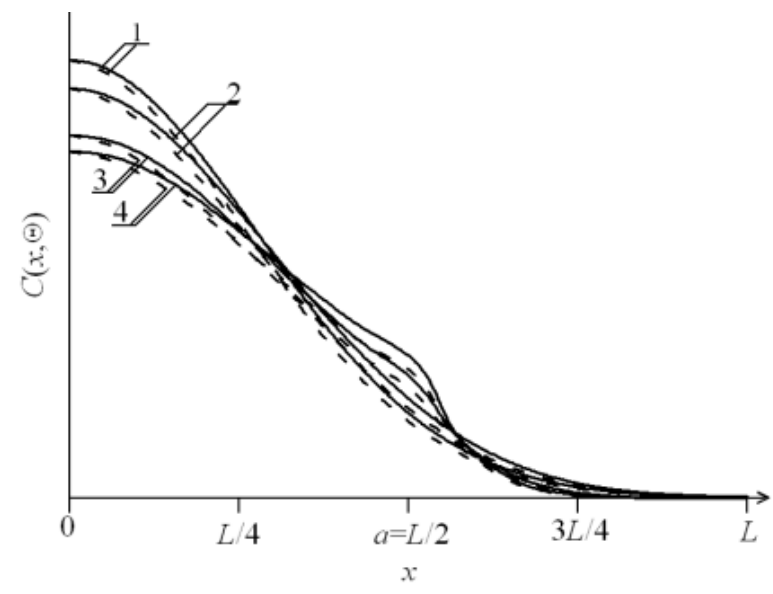

Figure 2. Distributions of infused dopant in heterostructure with two layers for different values of difference between dopant diffusion coefficients in layers of the heterostructure. Increasing of number of curves corresponds to increasing difference between values of dopant diffusion coefficients in layers of the heterostructure. Solid lines correspond to distributions of dopant without accounting adsorption of dopant. Dashed lines correspond to distributions of dopant with account adsorption of dopant 


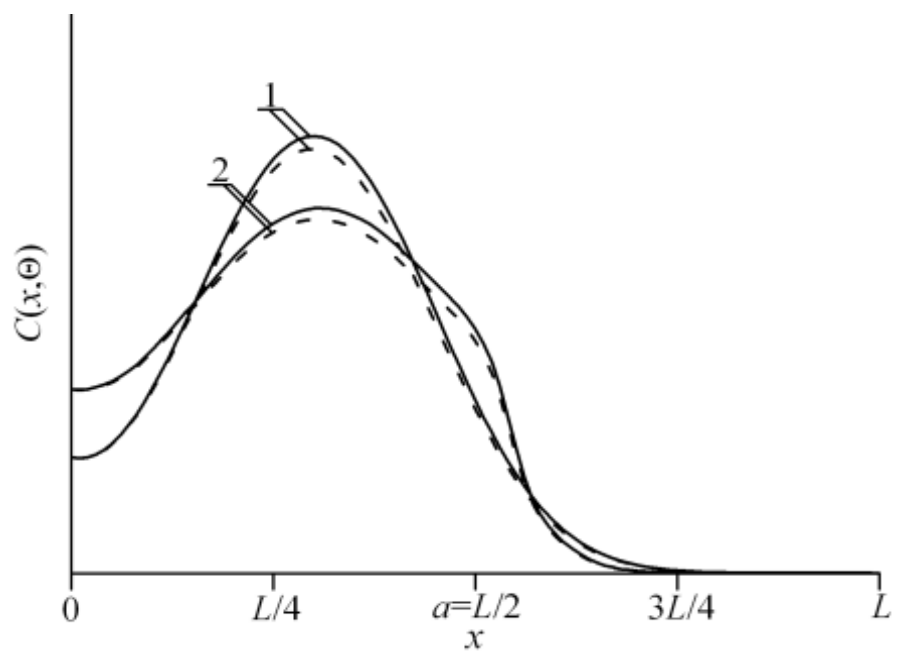

Figure 3. Distributions of implanted dopant in heterostructure with two layers for different values of difference between dopant diffusion coefficients in layers of the heterostructure. Increasing of number of curves corresponds to increasing difference between values of dopant diffusion coefficients in layers of the heterostructure. Solid lines correspond to distributions of dopant without accounting adsorption of dopant. Dashed lines correspond to distributions of dopant with account adsorption of dopant

$$
U=\frac{1}{L} \int_{0}^{L}[C(x, \Theta)-\psi(x)] d x
$$

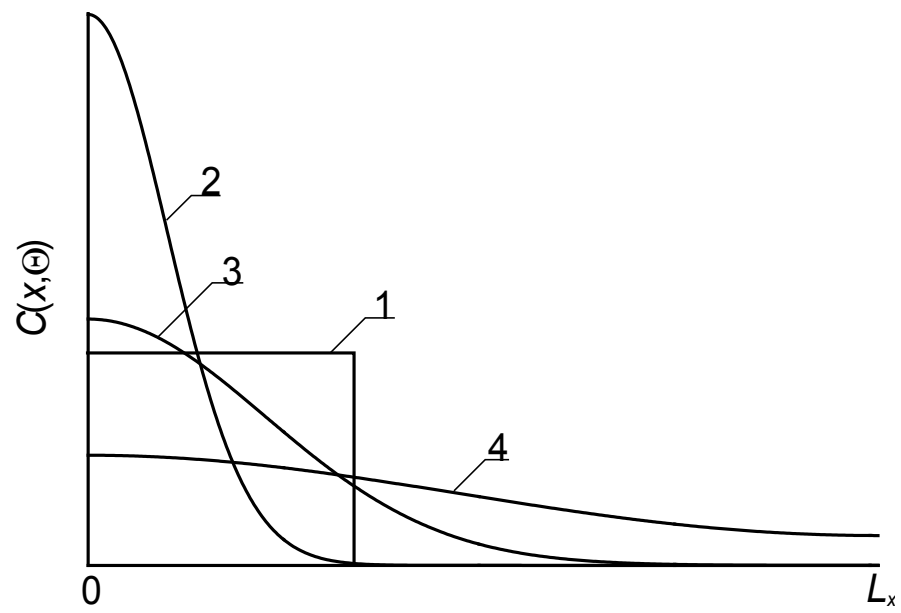

Figure 4. Spatial distributions of dopant in heterostructure with two layers for diffusion doping. Curve 1 is idealized distribution of dopant. Curves 2-4 are real distributions of dopant for different values of annealing time (increasing of number of curves corresponds to increasing of value of annealing time)

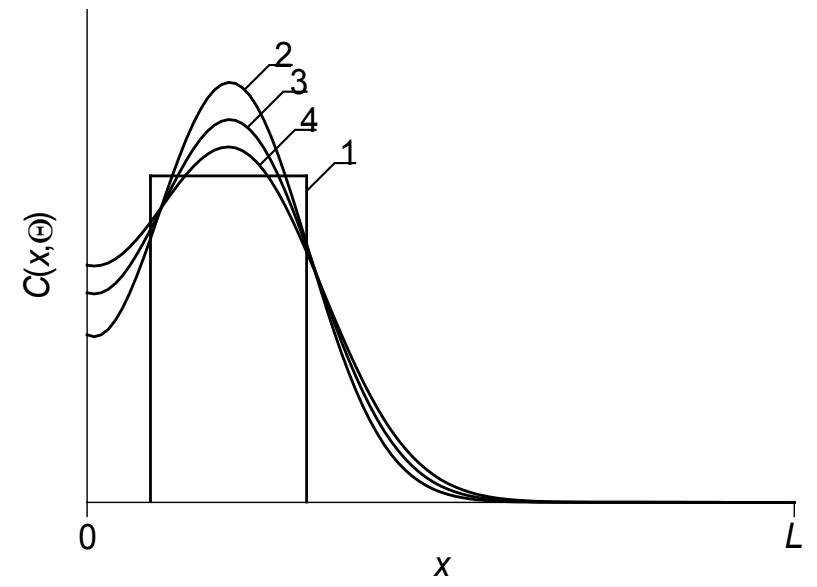

Figure 5. Spatial distributions of dopant in heterostructure with two layers for ion implantation. Curve 1 is idealized distribution of dopant. Curves $2-4$ are real distributions of dopant for different values of annealing time (increasing of number of curves corresponds to increasing of value of annealing time) 
where $\psi(x)$ is the approximation function, which was indicated as curve one on Figs. 4 and 5. It should be noted, that optimization of annealing time of implanted dopant attracted an interest, when the dopant distribution did not achieved interface between layers of heterostructure after annealing of radiation defects. In this case it should be done additional annealing of implanted dopant after annealing of radiation defects. Dependences of compromise annealing time on parameters are presented in Fig. 6 and 7.

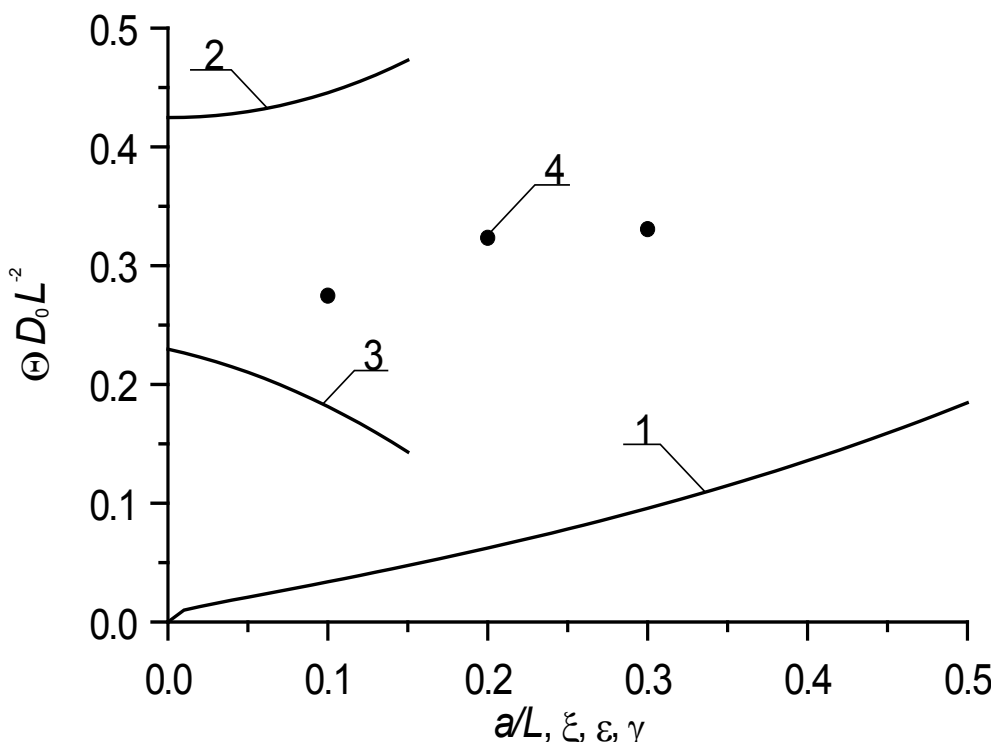

Figure 6. Dependences of dimensionless optimal annealing time of infused dopant, which have been calculated by minimization the mean squared error on several parameters. Curve 1 is the dependence of optimal annealing time on the ratio $a / L$ for pairwise equality of dopant diffusion coefficients and $\xi=\gamma=$ $\varepsilon=0$. Curve 2 is the dependence of optimal annealing time on the relation $\varepsilon=1-D_{1} / D_{2}$ for $a / L=1 / 2, \xi=\gamma=0$. Curve 3 is the dependence of optimal annealing time on the parameter $\xi$ for pairwise equality of dopant diffusion coefficients and $a / L=1 / 2, \gamma=\varepsilon=0$. Curve 4 is the dependence of optimal annealing time on the parameter $\gamma$ for pairwise equality of dopant diffusion coefficients and $a / L=1 / 2, \xi=\varepsilon=0$

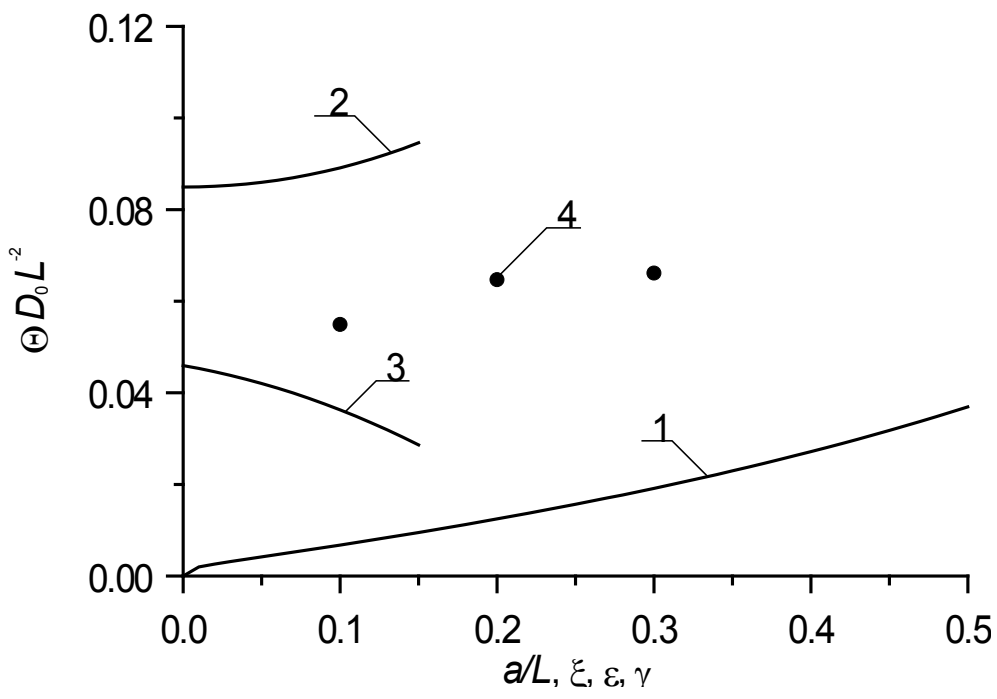

Figure7. Dependences of dimensionless optimal annealing time of implanted dopant, which have been calculated by minimization the mean squared error on several parameters. Curve 1 is the dependence of optimal annealing time on the ratio $a / L$ for pairwise equality of dopant diffusion coefficients and $\xi=\gamma=\varepsilon=0$. Curve 2 is the dependence of optimal annealing time on the relation $\varepsilon=1-D_{1} / D_{2}$ for $a / L=1 / 2, \xi=\gamma=0$. Curve 3 is the dependence of optimal annealing time on the parameter $\xi$ for pairwise equality of dopant diffusion coefficients and $a / L=1 / 2, \gamma=\varepsilon=0$. Curve 4 is the dependence of optimal annealing time on the parameter $\gamma$ for pairwise equality of dopant diffusion coefficients and $a / L=1 / 2, \xi=\varepsilon=0$ 


\section{Conclusion}

In this paper we introduce an approach to increase sharpness of implanted-junction and diffusive-junction heterorectifiers and at the same time to increase homogeneity of enriched by the infused or implanted dopants. We optimized annealing of dopant and/or radiation defects, which has been done during manufacturing the rectifiers. We also analyzed influence of adsorption of dopants on their distributions in $p-n$-junctions.

\section{Acknowledgments}

This work is supported by the contract 11.G34.31.0066 of the Russian Federation Government and educational fellowship for scientific research.

\section{REFERENCES}

[1] A. Kerentsev, V. Lanin, Power Electronics. Issue 1. P. 34 (2008).

[2] V.V. Vasil'ev, A.F. Kravchenko, Yu.P. Mashukov. Semiconductors. Vol. 36 (9). P. 1068-1073 (2002).

[3] A.V. Sakhorov, V.V. Lundin, E.E. Zavarin, M.A. Sinitsin, A.E. Nikolaev, S.O. Usov, V.S. Sizov, G.A. Mikhailovsky, N.A. Cherkashin, M. Hytch, F. Hue, E.V. Yakovlev, A.V. Lobanova, A.F. Tsatsul'nikov. Semiconductors. Vol. 43 (6). P. 841-846 (2009).

[4] J. Kundrotas, A. Cerskus, G. Valusis, L. H. Li, E. H. Linfield, A. Johannessen, E. Johannessen. J. Appl. Phys. Vol. 112 (4). P. 043105-043109 (2012).

[5] A. Kafar, S. Stanczyk, S. Grzanka, R. Czernecki, M.
Leszczynski, T. Suski, P. Perlin. J. Appl. Phys. Vol. 111 (8). P. 083106-083111 (2012).

[6] N.I. Volokobinskaya, I.N. Komarov, T.V. Matyukhina, V.I. Reshatnikova, A.A. Rush, I.V. Falina, A.S. Yastrebov. Semiconductors. Vol. 35 (8). P. 1013-1017 (2001).

[7] V.A. Gergel', Yu.V. Gulyaevb, M.N. Yakupov. Semiconductors. Vol. 39 (9). P. 1075-1079 (2005).

[8] I.G. Stamov, D.V. Tkachenko. Semiconductors. Vol. 42 (9). P. 1079-1085 (2008).

[9] Jih-Yuan Chang, Yen-Kuang Kuo. J. Appl. Phys. Vol. 112 (3). P. 033109-033114 (2012).

[10] E.L. Pankratov. Phys. Rev. B. Vol. 72 (7). P. 075201-075208 (2005).

[11] E.L. Pankratov. J. Appl. Phys. Vol. 103 (6). P. 064320-064330 (2008)

[12] E.L. Pankratov, E.A. Bulaeva. Int. J. Nanoscience. Vol. 11 (5). P. 1250028-1250035 (2012).

[13] E.L. Pankratov, E.A. Bulaeva. J. Comp. Theor. Nanoscience. Vol. 10 (4). P. (in press) (2013).

[14] Z.Yu. Gotra, Technology of microelectronic devices (Radio and communication, Moscow, 1991).

[15] E.I. Zorin, P.V. Pavlov and D.I. Tetelbaum, Ion doping of semiconductors (Moscow: Energiya, 1975).

[16] V.L. Vinetskiy, G.A. Kholodar', Radiative physics of semiconductors. ("Naukova Dumka", Kiev, 1979), (in Russian).

[17] P.M. Fahey, P.B. Griffin, J.D. Plummer. Rev. Mod. Phys. 1989. V. 61. № 2. P. 289-388.

[18] Yu.D. Sokolov. Applied Mechanics. Vol.1 (1). P. 23-35 (1955).

[19] E.L. Pankratov. Eur. Phys. J. B. Vol. 57 (3). P. 251-256 (2007). 